\title{
TTR
}

Traduction, terminologie, re?daction

\section{Amerika ou le corps du disparu}

\section{Monique Moser-Verrey}

Volume 5, numéro 2, 2e semestre 1992

Kafka pluriel : réécriture et traduction

URI : https://id.erudit.org/iderudit/037127ar

DOI : https://doi.org/10.7202/037127ar

Aller au sommaire du numéro

Éditeur(s)

Association canadienne de traductologie

ISSN

0835-8443 (imprimé)

1708-2188 (numérique)

Découvrir la revue

Citer cet article

Moser-Verrey, M. (1992). Amerika ou le corps du disparu. TTR, 5(2), 171-193.

https://doi.org/10.7202/037127ar

Tous droits réservés @ TTR: traduction, terminologie, rédaction — Les auteurs, 1992

Ce document est protégé par la loi sur le droit d'auteur. L'utilisation des services d'Érudit (y compris la reproduction) est assujettie à sa politique d'utilisation que vous pouvez consulter en ligne.

https://apropos.erudit.org/fr/usagers/politique-dutilisation/ 


\section{Amerika ou le corps du disparu*}

\section{Monique Moser-Verrey}

En 1988, Bernard Lortholary proposait une nouvelle version du fragment de roman que Kafka appelait Der Verschollene', basée sur l'édition critique de 1983 et intitulée Amerika ou le disparu'. Il s'agit en effet de l'Amérique $e^{3}$, roman connu en français grâce à la traduction d'Alexandre Vialatte depuis 1946, sous le titre et dans la forme que lui avait donnés Max Brod, lors de sa première publication en 1927. La version de Vialatte, amplement annotée par Claude David, qui propose pour titre l'Oublié plutôt que l'Amérique, se trouve également dans la bibliothèque de la Pléiade 4 . On dispose donc aujourd'hui de trois versions françaises d'un roman auquel Kafka travailla en 1912 et en 1914 puis abandonna à la faveur d'autres projets.

1. Franz Kafka, Der Verschollene. Herausgegeben von Jost Schillemeit (Frankfurt am Main, S. Fischer Verlag, 1983), Schriften Tagebucher Briefe Kritische Ausgabe.

2. Franz Kafka, Amerika ou le disparu (Paris, Flammarion, coll. «GF», 501, 1988).

3. Franz Kafka, l'Amérique (Paris, Gallimard, coll. «Folio», 406, 1965).

4. F. Kafka, Eusures complètes, vol. I (Paris, Gallimard, 1976), pp. 1 et suiv. 
Si j'intitule cette étude «Amerika ou le corps du disparu», c'est pour mettre en question un lieu commun des études kafkaïennes selon lequel Kafka, «ennemi de la chairs ${ }^{5}$ aurait répudié le corps et aurait même cherché à le faire disparaître au profit d'une érotisation de l'écriture $^{6}$. Mais qu'en est-il au juste de la disparition du corps dans les récits de Kafka? On trouve, en effet, dans la Métamorphose, par exemple, une image saisissante de la disparition du corps de Gregor Samsa qui se voit transformé en cafard, s'amenuise et finit par disparaître dans une poubelle. Georg Bendemann, le héros du Verdict, court lui aussi à sa perte, se laissant finalement tomber dans le vide. Il n'est donc pas étonnant que la carrière de Karl Rossmann, esquissée à la même époque, ne soit guère plus heureuse que celles de Gregor et de Georg. Cet anti-héros est également censé disparaître, comme l'indique d'ailleurs le titre du roman. Il s'agit là d'un destin commun aux trois «fils» ${ }^{7}$, mais il serait faux de croire que leur disparition ultime empêche les corps des protagonistes d'être présents dans le texte.

Mon propos sera donc d'analyser diverses modalités de la présence du corps de Karl dans le premier fragment de roman rédigé par Kafka, d'étudier la fonction de cette présence et de voir dans quelle mesure les versions françaises rendent adéquatement compte de quelques moyens stylistiques mis en cuvre par l'auteur pour faire parler ce corps. Kafka décrit en effet très peu l'aspect physique de ses personnages, mais il est attentif à leurs postures, leurs gestes, leurs mimiques, leurs regards et leurs déplacements, de sorte que leur corps investit l'espace de façon significative, tandis que leur regard et leur souffle rejoignent tous les horizons. La question est de savoir si cette manière particulière de représenter les corps gomme vraiment leur entité

5. Claude David, «la Fortune de Kafka», in F. Kafka, Euvres complètes, p. 13.

6. «Das Verschwinden des Körpers», in Detlef Cremer, Kafka: Die Erotik des Schreibens (Frankfurt am Main, Athenäum Verlag, 1989), pp. 135-140.

7. Kafka avait en effet pensé publier ensemble le Verdict, la Métamorphose et le Soutier, premier chapitre de l'Amérique, dans un recueil qu'il voulait intituler les Fils. 
sensuelle, voire érotique. Qu'un repérage systématique de l'inscription du corps de Karl dans le roman permette maintenant d'en juger.

\section{Le signalement de Karl}

Amerika ou le disparu met en scène un grand nombre de personnages qui ne portent pas tous un nom et, bien souvent, les noms existants ne sont même pas cités dans le texte. Les personnages sont plutôt identifiés selon leur âge, leur sexe, leur taille, leur nationalité, un lien de parenté, un emploi ou quelque autre fonction sociale. De même, les corps de ces personnages possèdent peu de traits distinctifs personnels, même si toutes les parties du corps sont abondamment nommées dans le texte, de la pointe des pieds au bout des cheveux. Un compte approximatif des quarante et quelques termes détaillant le corps des personnages révèle que les mains sont mises en jeu beaucoup plus souvent que les autres parties du corps. Les bras, la tête, le visage et les yeux sont aussi évoqués relativement souvent. Or, ces parties du corps sont en fait les principaux supports de la communication non verbale, et plus précisément les émetteurs de signes corporo-visuels cinétiques rapides tels les jeux de regards, les mimiques et les gestes ${ }^{8}$. Ces quelques indications, purement statistiques, permettent déjà de supposer que dans ce roman les corps sont essentiellement interactifs ${ }^{9}$ de même que les personnages s'insèrent dans un système relationnel plutôt que de se définir par ce qui leur serait propre. Selon la formule pertinente de Jean Starobinski, «les personnages de Kafka ne parviennent pas plus à avoir qu'à être» ${ }^{10}$; par contre, ils évoluent.

8. Catherine Kerbrat-Orecchioni, les Interactions verbales (Paris, Armand Colin, 1990), pp. 137-138.

9. Le vrai contexte de toute communication interpersonnelle est celui de l'interaction pour tous les chercheurs de la nouvelle communication présentés par Yves Winkin in Bateson, Birdwhistell, Goffman, Hall, Jackson, Scheflen, Sigman, Watzlawick, la Nouvelle Communication (Paris, Seuil, 1981), p. 24.

10. Jean Starobinski, «le Rêve architecte (à propos des intérieurs de Franz Kafka)», Lettres, 23 (Genève, 1947), pp. 31-32. 
Voyons d'abord ce que l'on sait de Karl Rossmann, le disparu, arrivé à New-York à bord d'un bateau allemand pour vivre en Amérique une série de déboires dont personne ne connaîtra la fín. Les premiers mots du roman disent sa jeunesse. Il n'a que seize ou dix-sept ans ${ }^{11}$ et peut donc être considéré comme un frère cadet des autres héros romanesques de Kafka, plus sobrement appelés K. La critique souligne depuis longtemps la naïveté enfantine de Karl, compensée par une grande acuité visuelle ${ }^{12}$. En effet la première phrase du roman nomme doublement son regard: «[...] erblickte er die schon längst beobachtete Statue der Freiheitsgöttin wie in einem plötzlich stärker gewordenen Sonnenlicht. Ihr Arm mit dem Schwert ragte wie neuerdings empor $[\ldots] \gg(7)^{13}$. Curieusement, cette insistance sur le regard de Karl, sensible dans l'accumulation des verbes erblicken (apercevoir) et beobachten (observer), n'est retenue dans aucune des versions, car celles-ci accordent à la statue de la Liberté la fonction de sujet de la proposition. Dès lors, c'est elle qui «apparaît» (V.11) ${ }^{14}$ ou «paraît» (L.17) ${ }^{15}$ à Karl plutôt que d'être aperçue par lui: «[...] la statue de la Liberté, qu'il observait depuis longtemps, lui apparut dans un sursaut de lumière. On eût dit que le bras qui brandissait l'épée s'étant levé à l'instant même [...]» (V.11). «[...] la statue de la Liberté qu'il regardait depuis un long

11. Les éditions courantes du roman donnent seize ans à Karl, tandis que l'édition critique et à sa suite, Amerika ou le disparu, rétablissent les dix-sept ans que l'on a dans le manuscrit.

12. Wolfgang Jahn, Kafkas Roman «Der Verschollene» («Amerika»), (Stuttgart, Metzlersche Verlagsbuchhandlung, 1965), p. 104.

13. Toutes les citations allemandes du texte, suivies du numéro de la page de référence, renvoient à l'édition critique de Jost Schillemeit, Franz Kafka, Der Verschollene.

14. Les références à la traduction de Vialatte sont incluses dans le corps du texte. Précédées de l'initiale du traducteur, elles renvoient à Franz Kafka, l'Amérique.

15. Les références à la traduction de Lortholary sont incluses dans le corps du texte. Précédées de l'initiale du traducteur, elles renvoient à Franz Kafka, Amerika ou le disparu. 
moment lui parut tout d'un coup éclairée d'un soleil plus vif. Son bras armé d'un glaive semblait brandi à l'instant même [...]» (L.17).

Cette transformation, qui renverse les rapports entre sujet et objet, s'imposait sans doute à cause de la difficulté qu'il y a à rendre en français le wie allemand. La stylistique comparée classe, en effet, le mot wie, adverbe et conjonction, parmi les éléments intraduisibles de la langue allemande ${ }^{16}$. Or la fascination qu'exerce sur Karl la statue de la Liberté, dont le texte original souligne la divinité, Freiheitsgöttin, est doublement signalée par ce wie qui déréalise et la perception de la lumière et celle du temps. Tout se passe pour lui comme si l'éclairage s'intensifiait subitement et que le bras de cette déesse brandissait tout aussi subitement un glaive. La traduction de Vialatte souligne la surréalité de cette vision en négligeant d'une part la modalisation de l'effet de lumière, car ce qu'il appelle "un sursaut de lumière» est donné pour objectif, et en dénonçant d'autre part, dans une note du traducteur, l'irréalité notoire du glaive substitué au flambeau de «la vraie statue». Dans la version de Lortholary les verbes «paraître» et «sembler» ramènent ces effets étonnants à une question de perspective. Il s'agit bien de la façon dont le héros voit le monde, plutôt que de phénomènes susceptibles de frapper un chacun, comme le suggère la locution «on eût dit» choisie par Vialatte. Maja Goth a très justement souligné que la première réception de Kafka en France s'est faite sous le signe du surréalisme ${ }^{17}$ dont les options ne sont pas sans infléchir visiblement les choix stylistiques du premier traducteur.

Si le regard de Karl constitue le premier mode d'inscription de son corps dans le texte, sa force juvénile est également mentionnée dès le départ, lorsqu'il hisse sa valise sur son épaule. C'est un «solide garçon» (V.11) ou un "garçon robuste» (L.17). Mais il est aussi un «beau garçon» (V.21) ou un «joli garçon» (L.26) selon l'avis malicieux de Line, une des filles de cuisine du bateau. Sa stature est enfin mentionnée dans une lettre que Johanna Brummer, sa séductrice, a

16. Alfred Malblanc, Stylistique comparée du français et de l'allemand (Paris, Didier, 1968), p. 117.

17. Maja Goth, Franz Kafka et les lettres françaises (1928-1955) (Paris, Librairie José Corti, 1956), p. 242. 
adressée au sénateur Jakob, un oncle maternel de Karl vivant à NewYork. Celui-ci vient retrouver son neveu sur le bateau à peine arrivé et le reconnaît grâce au signalement donné dans cette lettre, signalement dont il a noté «les traits les plus marquants» (L.46) dans son calepin. D'entrée, le texte insiste donc sur le «signalement» (L.42) de Karl, mais le lecteur attentif n'aura pas l'avantage d'en prendre connaissance. Nous avons affaire ici à ce que Wolfgang Iser a identifié comme la structure d'appel des textes la plus efficace, à savoir le blanc (Leerstelle) ${ }^{18}$ permettant au lecteur de suppléer les détails tus et l'incitant par là-même à se faire sa propre image mentale du personnage.

Puisque le signalement de Karl n'est pas livré, son corps demeure potentiellement vivant. Aucune description ne le fige ${ }^{19}$ et de plus l'intrigue en interdit toute perception objective, parce qu'il est sans cesse évoqué comme le lieu d'un désir. Le mot de Line fait rire toutes les filles de cuisine en semant l'équivoque au sujet de la relation que le soutier, à qui il est adressé, peut bien entretenir avec ce «joli garçon». Le signalement, donné par Johanna Brummer, est écrit selon les termes de $M$. Jakob «avec une roublardise un peu simple, mais sans malice, et beaucoup d'amour» (L.42). Ces observations, qui ne sont «naturellement pas d'une exactitude policière» (L.46), donnent finalement bien du plaisir à l'oncle lui-même, puisqu'il les confronte avec la figure de Karl «par jeu» et «dans le seul but de se distraire» (L.46). Il n'y a sans doute rien de plus agréable que tout ce qui concerne le corps du disparu, mais les détails en sont réservés aux intimes et Karl lui-même n'aura accès aux descriptions de sa séductrice que «dans le silence de la chambre qui l'attend» (L.43). Tout se passe finalement comme si tant de perceptions érotisées du corps de Karl ne laissaient pas de place à une vision neutre de son apparence qui pourrait être donnée une fois pour toutes. Cette apparence demeurera au contraire un centre d'intérêt tout au long du roman.

18. Wolfgang Iser, Der Akt des Lesens (Munchen, W. Fink, 1976), pp. 284 et suiv.

19. Le danger de la «pétrification descriptive» dont les romanciers doivent «se dépêtrer» est signalé par Raymonde Debray Genette dans Métamorphoses du récit (Paris, Seuil, 1988), p. 221. 
La scène des retrouvailles avec l'oncle d'Amérique a lieu en présence d'un grand nombre de personnages dont Karl devient bientôt le point de mire (L.45). Tout le monde regarde Karl dans ce roman, on s'attroupe autour de lui pour l'observer et même New-York le regarde «avec les cent mille fenêtres de ses gratte-ciel» (L.27). Le voilà obligé de se donner une contenance. Pour présenter son neveu, l'oncle Jakob explique publiquement qu'il a été chassé de chez ses parents pour avoir fait un enfant à la bonne, mais il se garde bien de donner lecture de la lettre qu'il a reçue de la Brummer. Puis il veut que sa parenté avec Karl soit généralement reconnue et sollicite alors l'aveu du neveu de même que les félicitations de l'assistance. Il s'ensuit une série de paroles et de gestes de circonstance qui établissent la nouvelle identité de Karl. Peu importe au fond le signalement du personnage, car son image publique et romanesque dépend ici comme ailleurs de son comportement.

La thêâtralité inhérente au traitement du personnage kafkaïen est un fait bien connu. Tandis que Wolfgang Jahn analyse la façon dont l'écriture du fragment Der Verschollene visualise les caractéristiques et les fonctions des personnages, ${ }^{20}$ Evelyn Torton Beck, dont les recherches ont mis en valeur l'impact du théâtre yiddish sur le style de Kafka, démontre combien leurs paroles et leurs actes sont chargés de sens. C'est sur ce plan-là que se réalise une individualisation qui doit compenser le signalement et parfois le nom laissés en blanc ${ }^{21}$. Dans Amerika ou le disparu elle note six véritables scènes parmi lesquelles les retrouvailles de Karl et de son oncle figurent en première place. Or le lecteur de cette scène se trouve essentiellement dans la même position que les témoins des retrouvailles qui regardent la scène. Il ne peut pas se reporter au signalement de Karl, mais il peut apprécier ce qu'il dit et ce qu'il fait.

20. Wolfgang Jahn, Op. cit., pp. 32-67.

21. Evelyn Torton Beck, Kafka and the Yiddish Theater (Madison, Milwaukee and London, The University of Wisconsin Press, 1971), pp. 35-36. 


\section{La représentation du corps en interaction}

Voyons maintenant comment Kafka construit dans sa narration la simultanéité du verbe et du geste telle qu'elle se produit en situation d'interaction sociale ou théâtrale. Le rituel ${ }^{22}$ de reconnaissance entre les deux principaux protagonistes de la scène se lit ainsi: $\ll D u$ bist mein Onkel, sagte Karl und küßte ihm die Hand und wurde dafür auf die Stirn geküßft.» (p. 43) Il apparaît clairement que les éléments verbaux et les éléments gestuels de cet aveu public sont simplement juxtaposés et reliés par la conjonction und (et). Cette stratégie communicative se retrouve en effet partout où le texte représente une interaction entre personnages. Or les versions ne reproduisent pas cette sorte de syntaxe. La stylistique comparée signale depuis longtemps que l'allemand fait un usage beaucoup plus fréquent de la conjonction de coordination und que le français n'utilise le «et». Cette constatation suggère que les rapports logiques selon lesquels des unités phrastiques sont agencées restent plus volontiers implicites en allemand qu'en français. ${ }^{23}$

Vialatte a donc recours au gérondif suivi d'une subordonnée relative: «Tu es mon oncle, dit Karl en lui baisant la main, ce qui lui valut un baiser sur le front» (V.43). Lortholary opère la même transformation puis coordonne les deux gestes en sacrifiant par contre l'élément de réciprocité marqué par l'adverbe dafür: «Tu es mon oncle, dit Karl en lui baisant la main et en recevant un baiser sur le front» (L.44). Par l'emploi du gérondif les versions françaises font apparaître le geste comme une circonstance accompagnant l'acte de parole ${ }^{24}$. Cette option n'est d'ailleurs pas entièrement étrangère à la syntaxe de

22. Ce terme est à entendre ici dans le sens profane que lui donne Erving Goffman, qui étudie justement la vie sociale sous l'aspect de sa théâtralité. Voir, entre autres, la Mise en scène de la vie quotidienne. 2: Les Relations en public (Paris, Minuit, 1973), p. 69.

23. Alfred Malblanc, Op. cit., p. 191.

24. "Le gérondif est la forme adverbiale du verbe: comme l'adverbe, en effet, il exprime certaines circonstances de l'action marquée par un autre verbe de la phrase.» (Maurice Grevisse, le Bon usage: grammaire française, Gembloux, J. Duculot, 1961, par. 799.) 
Kafka. Le geste qui accompagne les félicitations du capitaine se présente bien sous la forme d'un complément circonstanciel de manière: «Ich bin glücklich, sagte der Kapitän mit einer Verbeugung, wie sie nur militärisch geschulte Leute zustandebringen, Ihren Neffen, Herr Senator, kennengelernt zu haben» (44). Là encore le gérondif est la seule solution envisagée par les traducteurs qui rendent mit einer Verbeugung par «en s'inclinant» (V.43) et «en inclinant le buste» (L.44). Il faudra voir en quoi ces légers écarts de construction, qui instaurent un rapport logique plus explicite entre la parole et le geste que ne le fait la simple addition des données, modifient la représentation romanesque de l'interaction.

Parmi les registres non verbaux de l'interaction humaine on distingue généralement les regards des mimiques, les mimiques des gestes et les gestes des postures, qui à leur tour donnent à voir des rapports proxémiques chargés de sens. Mais tous ces éléments se combinent, comme on l'a vu, avec du discours direct dans le texte. Si on isole les mimiques de Karl, on s'aperçoit qu'une fois sur deux il sourit. Il s'agit donc d'une mimique simplement conventionnelle qui se combine avec des regards, des gestes ou des paroles de circonstance. Cette mimique est alors généralement notée en passant au participe présent lachend ou lächelnd. À quelques occasions les mines de Karl sont plus éloquentes. On lui voit le visage sérieux dans sa fonction de liftier, des larmes dans les yeux au piano de Klara, les dents serrées au moment de l'embauche et enfin la tête rouge dans la loge du portier en chef qu'il déteste. Ce sont surtout des gestes et des postures qui viennent s'ajouter aux répliques de dialogues dans des propositions coordonnées par und. Le français reprend sans problème la même formule, si le und introduit une nouvelle phrase: ««Comment s'appelle-t-il donc?» Et d'une poche secrète[...] il tira une carte de visite» (L.19). Dans certains dialogues, Lortholary va jusqu'à éviter l'incise sagte er «dit-il», pour pouvoir enchaîner de cette façon-là. «"Wollen Sie also das Geld unter dieser Bedingung? " fragte Karl und griff in die Westentasche» (212). ««Eh bien, est-ce qu'à cette condition vous voulez l'argent?» Et Karl, en posant cette question, mit la main à la poche de son gilet» (L.175). Le plus souvent cependant, c'est le gérondif qui l'emporte. 
Voici, parmi tant d'autres, un exemple qui illustre particulièrement bien le fait que la recherche d'intimité de Karl se présente en allemand par l'enchaînement d'un déplacement et d'un geste, tandis qu'en français ces mêmes éléments constituent plutôt le cadre dans lequel la réplique se donne.

"Ich hätte eine Bitte, die Sie nicht mißverstehen dürfen» sagte Karl, ging eiligst zu Herrn Pollunder hin und legte, um ihm recht nahe zu sein, die Hand auf die Armlehne des Fauteuils. (104)

«J'aurais une demande à vous faire, mais je ne voudrais surtout pas que vous vous mépreniez sur sa signification, dit Karl en allant droit à $M$. Pollunder et en mettant la main sur le bras de son fauteuil pour être tout près de lui.» (L.91)

Il est évident qu'en évitant la forme conjuguée du verbe la version donne de l'ensemble des éléments de l'interaction une représentation plus homogène, tandis que le texte original souligne davantage le dynamisme du personnage par le cumul de verbes à la troisième personne du singulier.

S'il est vrai que les personnages se définissent par leurs gestes, cette définition est d'autant plus précise que la spécificité de ces gestes est souvent expliquée. Ainsi, lors de la scène des retrouvailles, le capitaine s'incline «comme seuls savent le faire les gens qui ont eu une formation militaire» (L.44). Le soutier, qui félicite également Karl, lui serre la main «voulant traduire par là quelque chose comme de la reconnaissance» (V.44). Et pour sa part le sénateur évite cette sorte de contact en reculant «comme si le soutier outrepassait ses droits» (L.45).

Les formules explicatives varient. Elles peuvent être objectives et faire apparaître un trait culturel donné, renvoyant aux rituels d'une institution connue, comme l'armée en l'occurrence. Mais elles peuvent aussi être subjectives et révéler les émotions du personnage concerné, ici son admiration ou sa reconnaissance. Souvent, cependant, elles sont purement spéculatives, livrant quelques éléments d'interprétation sur le mode incertain de la comparaison conditionnelle. $Y$ avait-il vraiment outrecuidance de la part du soutier à vouloir présenter ses hommages au sénateur? Le sénateur voulait-il vraiment éviter cet échange? Tout en 
notant les mouvements de ces deux personnages, le texte n'en retient l'explication qu'à titre d'hypothèse.

Ce moyen stylistique permettant de creuser les mobiles de l'interaction tout en étudiant les jeux du corps est, dit-on, emprunté à Charles Dickens. De l'aveu même de Kafka son roman américain doit beaucoup au David Copperfield de l'auteur anglais. Hartmut Binder a relevé dans ces deux cuvres de nombreux exemples d'une utilisation très semblable de la proposition comparative conditionnelle, mais il semble évident que Kafka l'a davantage exploitée, chargeant volontiers cette subordonnée d'informations nouvelles, tandis que Dickens se contente d'indications redondantes ${ }^{25}$. Binder a sans doute raison de ramener l'utilisation que fait Kafka de la comparaison conditionnelle à une question de perspective narrative, mais le procès qu'il fait à ceux qui ont souligné l'irréalité ou plutôt la surréalité des éléments proposés dans le terme comparant n'est pas entièrement fondé. ${ }^{26}$

Comme Karl est le principal focalisateur à l'intérieur du monde de la diégèse, ce sont généralement les faits et gestes des autres personnages, tels l'oncle Jakob et le soutier, par exemple, qui sont notés et mis en rapport avec les intentions, les raisons ou les émotions de ceux-ci. Présentés ainsi dans la perspective de Karl, les mobiles de ces personnages ne peuvent pas être des faits avérés. Il s'agit plutôt de suppositions et d'interprétations plus ou moins justes dont le narrateur abandonne au personnage la responsabilité. Voilà pourquoi le als allemand est suivi du mode subjonctif, tandis qu'on trouve des propositions conditionnelles introduites par if ou si en anglais et en français. Cette technique narrative est voisine du discours indirect libre qui laisse également en suspens la vérité objective des faits rapportés, sans qu'il soit pour autant question de surréalité. Mais l'incertain probable peut fort bien glisser du côté de l'irréel et du surréel, lorsqu'on a affaire à des fantasmes ou des rêves de Karl. Avant de s'endormir sur le canapé de Brunelda, il songe, par exemple, à l'empressement avec

25. Hartmut Binder, Kafka in Neuer Sicht. Mimik, Gestik und Personengefüge als Darstellungsformen des Autobiographischen (Stuttgart, J. B. Metzler, 1976), p. 195.

26. Ibid., pp. 198 et suiv. 
lequel il s'aquitterait de ses tâches, s'il pouvait trouver un meilleur emploi. Et le narrateur de préciser: «Ces bonnes résolutions se pressaient dans sa tête comme si son futur chef avait été debout devant le canapé et les avait lues sur son visage.» (L.283) Ici ce n'est plus le langage du corps qui suscite des spéculations sur les dispositions intérieures du personnage, mais au contraire les dispositions intérieures de Karl qui font naître une scène explicative parfaitement surréelle où apparaît un chef imaginaire qui saurait justement reconnaître ces bonnes intentions en scrutant son visage.

Le surréel fait également irruption dans la proposition comparative conditionnelle, lorsque les gestes observés dérangent Karl. On peut même reconnaître là le germe de métaphores animales qui prennent plus d'ampleur dans certains récits de Kafka. Ainsi, un employé voulant empêcher Karl de s'approcher du caissier principal du navire le poursuit «penché en avant et les bras écartés pour l'attraper, comme s'il poursuivait un insecte» (L.28) et la femme de l'auberge, qui jette à deux mains les affaires de Karl dans sa mallette, le fait comme s'il s'agissait de «réduire des animaux récalcitrants à se coucher à ses pieds» (V.125). Il y a sans doute un rapport entre la crainte et la mauvaise humeur que ces gestes produisent sur le héros et la comparaison surréelle qu'ils inspirent au narrateur.

La comparaison conditionnelle ne constitue pas une difficulté de traduction particulière. Deux fois sur trois le als suivi du subjonctif allemand est rendu par «comme si» en français. Une comparaison nominale ou une phrase infinitive introduite par «comme pour» sont parfois préférées pour leur concision, quoique la dimension hypothétique du terme comparant se perde alors. Cette dimension incertaine du propos peut finalement être aussi indiquée par le verbe sembler ou l'expression «on eût dit» déjà observés plus haut comme moyens de rendre le wie allemand. Il apparaît donc que la comparaison et la comparaison conditionnelle entament la solidité des évidences physiques observées en les liant à des explications subjectives, susceptibles de devenir purement spéculatives ou visionnaires et d'ouvrir ainsi la porte au fantastique. 


\section{Le regard de Karl}

D'une part, Karl est entouré de regards bienveillants, admiratifs, amoureux, curieux ou hostiles; d'autre part, il est lui-même le principal focalisateur du roman. Son corps est donc bien souvent évoqué sous la modalité du regard. Or, le regard d'un personnage non seulement donne à voir le monde sous un angle subjectif, mais encore s'inscrit dans les réseaux de l'interaction, puisque ses mouvements constituent l'un des registres de la communication non verbale. Il importe donc d'être attentif à la façon dont Karl oriente ses regards, cherche ou évite ceux des autres, insiste ou glisse sur un contact, et ainsi de suite, pour mieux le comprendre.

La langue allemande offre un dispositif impressionnant pour noter l'orientation des regards de même que leur intensité. À travers une soixantaine de passages qui concernent les regards de Karl, nous avons répertorié une vingtaine d'expressions modulant la spécificité et l'orientation de ces regards. Il s'agit essentiellement des verbes sehen, schauen et blicken (voir, regarder) démultipliés par l'addition de particules qui spécifient la direction et la modalité de la vision. Ainsi, il y a ansehen, aufsehen, umsehen, zusehen, hinsehen, mais aussi hinaussehen ou hinübersehen en plus de sehen. En plus de schauen, on trouve aussi anschauen, ausschauen, herüberschauen, hindurchschauen; en plus de blicken, erblicken, überblicken, hinüberblicken, sans compter les références nominales aux regards: Blick, Blicke, Seitenblick et les verbes moins neutres comme beobachten (observer) ou starren (fixer).

Il ne peut pas être question de faire ici une analyse exhaustive de ce que la qualité des regards de Karl révèle au sujet de ses rapports avec le monde, ni de la façon dont les traducteurs rendent ou évitent en français les précisions de l'expression allemande. Nous nous en tiendrons à quelques exemples frappants qui montrent comment Karl cherche ou refuse le contact visuel avec autrui ou avec le monde et comment l'orientation de son regard reflète ses états d'âme, ses aspirations, et articule finalement son destin.

En début de parcours, il y a une occasion où le regard de Karl est consciemment mis en jeu pour obtenir certains effets. C'est précisément le cas lorsque le soutier prononce un réquisitoire confus 
contre son ennemi, Schubal, dans l'espoir de pouvoir conserver son emploi à bord du navire.

Karl fixa alors le commandant de toutes ses forces, d'un air de connivence, comme un confrère, afin qu'il ne se laisse surtout pas influencer défavorablement par ce propos assez maladroit. (L.31)

La version de Vialatte est plus concise:

À cet endroit, Karl regarda fixement le capitaine d'un air d'intelligence, en confrère, pour l'empêcher de se laisser prévenir contre le chauffeur par la maladresse du discours. (V.27)

En fait les deux traducteurs ont resserré la phrase allemande qui est nettement la plus longue:

Bei dieser Stelle starrte Karl mit aller Kraft den Kapitän an, zutunlich, als sei er sein Kollege, nur damit er sich durch die etwas ungeschickte Ausdrucksweise des Heizers nicht zu dessen Ungunsten beeinflussen lasse. (25)

On peut admirer le choix de l'adverbe «alors» qui permet à Lortholary de faire l'économie de «À cet endroit»; on peut s'étonner que Vialatte ait osé omettre "de toutes ses forces», mais l'un et l'autre étendent l'expression verbale allemande zutunlich anstarren, qui ne concerne que le regard, à une vision plus globale de «l'air d'intelligence» ou «l'air de connivence» du personnage. Voilà qui souligne combien les différents registres de la communication non verbale ceuvrent de concert et peuvent se substituer les uns aux autres sans fausser la perception d'une interaction particulière. Ainsi, «regarder d'une certaine manière» se traduit ici par «se donner tel ou tel air».

La présomption de Karl est cependant à la fois mieux explicitée et mieux excusée dans l'original allemand que dans les traductions. La subordonnée «comme s'il était son collègue» dénote que Karl fait semblant d'être le collègue du capitaine, tandis que les syntagmes nominaux «en confrère» et «comme un confrère» ne laissent percevoir 
la supercherie que de façon implicite. Aussi les phrases françaises se dispensent-elles de relever le nur allemand qui précise que ce regard manipulateur avait pour seul but de venir en aide au soutier. L'esprit de hiérarchie qui commande ici les subtilités de l'explication d'un regard ne nous touche plus aujourd'hui de la même façon qu'il ne marquait la société pragoise du début du siècle dont l'habitus austro-hongrois imprègne l'Amérique de Kafka. Le traducteur pourrait donc invoquer plusieurs raisons pour en faire abstraction. Mais, comme cet esprit de hiérarchie est aussi présent dans plusieurs autres passages du roman, ${ }^{27}$ il semble plus conséquent de le maintenir partout où il s'exprime.

Dans la maison de campagne de $\mathrm{M}$. Pollunder, Karl est passablement humilié par la fille de son hôte, Klara, qui, après l'avoir renversé sur un canapé, menace de le gifler. Il rêve de n'avoir plus rien à faire avec elle et évite donc à tout prix son regard. La difficulté qu'il y a à rendre en français les particules d'orientation spatiale allemandes est évidente dans ce passage. Comment traduire hinaufstarren? «Il restait donc là, couché, les regards fixés sur le plafond» (V.86), dit Vialatte, tandis que Lortholary traduit: «Aussi restait-il étendu là, les yeux fixement levés» (L.82). Cette fois-ci la concision de l'original: «Und so lag er, starrte hinauf» (93) ne peut pas être sauvegardée. Il faut étoffer l'expression en français, à la rigueur même évoquer un point de repère spatial, le plafond, tout au plus implicite dans l'expression allemande.

Au fur et à mesure que sa situation sociale se détériore, le regard de Karl perd de son aplomb. Lorsqu'il s'aperçoit qu'il a perdu la photographie de ses parents - à moins que ce ne soient ses compagnons de route qui la lui aient volée -, il fait carrément pitié. «Aber das ist doch unmöglich, sagte Karl, und seine Hilfe suchenden Blicke zogen den Kellner näher.» (167) On retrouve ici la coordination si courante dans les phrases kafkaiennes entre le discours du personnage et l'expression non verbale qui l'accompagne. Aucun des traducteurs ne retient cette formule. $\grave{A}$ la simple juxtaposition des éléments, ils préferent l'articulation explicite, soit d'un rapport de causalité, soit d'un rapport temporel de simultanéité. On lira donc: «Mais c'est impossible,

27. Régine Robin, Kafka (Paris, Belfond, 1989), p. 196. 
dit Karl, dont le regard semblait chercher une aide, ce qui fit rapprocher le garçon» (V.150); ou encore: «Mais enfin c'est impossible, dit Karl tandis que ses regards désemparés faisaient s'approcher le serveur.» (L.140)

La situation de Karl s'aggrave encore et il prend conscience de son incapacité à communiquer adéquatement du regard au moment où il se fait prendre en faute par ses supérieurs à l'Hôtel Occidental. La cuisinière en chef, sa protectrice, vient de lui rappeler, en présence du chef des grooms et du portier en chef, ses bonnes dispositions à son égard.

Mais Karl, au lieu de regarder la cuisinière en chef dont les yeux cherchaient certainement les siens, observait fixement le plancher bien que cette attitude ne pût qu'être mal interprétée. (V.214)

Conservant l'ordre de la phrase allemande, Lortholary écrit:

Quant à Karl, bien que ce pût être interprété comme un signe fâcheux, il ne regardait pas la cuisinière en chef, qui quêtait certainement son regard, il fixait le sol devant lui. (L.196)

Ici, ce sont les versions françaises qui cumulent les verbes évoquant le regard, tandis que l'original se contente d'une seule mention de blicken:

Karl aber blickte, obwohl das nur als schlechtes Zeichen aufgefaßt werden konnte, nicht auf die Oberköchin, die gewiß nach seinem Blick verlangte, sondern vor sich auf den Fußboden. (239)

La valeur sémiotique de l'orientation du regard est tout à fait explicite dans ce passage. 
Si Karl se montre incapable d'émettre le signe attendu, ce n'est pas par incompétence communicative, ${ }^{28}$ mais bien parce que le portier en chef lui a serré le bras si fort qu'il est tout occupé par cette douleur. D'ailleurs, le portier en chef possède l'art de le troubler, de sorte qu'il n'est plus maitre de ses mouvements qui finissent par dénoter le contraire de ce qu'il voudrait signifier. Voici comment Karl se comporte à l'égard de son tortionnaire après avoir aperçu le travail exténuant qu'il impose aux sous-portiers: «[...] oubliant presque que le portier en chef était son grand ennemi, il leva les yeux vers lui et hocha la tête en silence pour l'approuver» (L.211). Fidèle à lui-même Vialatte préfère: «il leva ses regards vers lui» (V.230). En fait, ces deux locutions correspondent au verbe allemand aufsehen.

\section{Les paramètres existentiels des jeux de regards}

Dans l'ensemble du roman aufsehen et hinuntersehen s'opposent comme le bonheur et le malheur, ou encore l'admiration et la confusion. D'entrée, l'oncle Jakob, qui tient à inculquer quelques bons principes à son neveu, lui cite le mauvais exemple de ceux qui perdent des journées entières à leur balcon «à regarder dans la rue comme des moutons égarés» (V.54) ou des «brebis égarées» (L.54). Ni l'une ni l'autre des versions ne spécifie que les regards sont baissés vers la rue. Cette orientation, sensible dans le syntagme allemand auf die Straße hinuntersehen, reste forcément implicite en français, de sorte que l'on perd l'opposition nette entre le haut et le bas, qui véhicule pourtant des contenus déclarés dans le texte. Lorsque Karl est très malheureux sur le balcon de Brunelda, on lit bien: «Traurig sah er auf die Gasse hinunter, als sei dort der Grund seiner Traurigkeit.» (322-323) «Puis il regarda mélancoliquement dans la rue comme si le motif de sa

28. La linguistique du discours postule dans ce sens une «compétence paralinguistique en plus de la compétence linguistique" (Catherine Kerbrat-Orecchioni, l'Énonciation: de la subjectivité dans le langage. Paris, Armand Colin, 1980, p. 19). La compétence communicative inclut en fait «l'ensemble des moyens, verbaux et non verbaux, mis en cuvre pour assurer la réussite de la communication» (A. Sinclair, «le Développement de la compétence communicative», in J. GérardNaef, dir., Savoir parler, savoir dire, savoir communiquer (Neuchâtel, Delachaux et Niestlé, 1987, p. 134). 
tristesse se trouvait là.» $(\mathrm{V} .286)^{29}$ Il est intéressant de noter que Lortholary conserve l'expression physique de la dépression en traduisant le regard par un geste et en étoffant l'adverbe de lieu: «Il se pencha tristement vers la rue, comme si la cause de sa tristesse était là-bas à ses pieds.» (L.261)

Le moment euphorisant du regard levé sert pour sa part de cadre à l'ensemble du fragment de roman. Pour Torton Beck l'image particulière de la statue de la Liberté, que Karl aperçoit brandissant un glaive, renvoie à celle des anges du grand thêatre d'Oklahoma et se situe très nettement, comme ceux-ci, dans la tradition juive à la suite d'une pièce yiddish de l'auteur Gordin. Kafka ne se serait-il pas amusé à fusionner cette gardienne du port de New-York avec l'image des anges montant la garde, glaive en main, devant les portes du jardin d'Eden ${ }^{30}$ ? Cette hypothèse est d'autant plus stimulante que, dans la perspective de Karl Rossmann, c'est surtout la hauteur de cette statue qui compte, comme d'ailleurs celle des anges du grand theâtre: «So hoch!» (7), «Si haute!» (L.17). Voilà sa première pensée, alors qu'il oublie de sortir du bateau et se fait pousser contre la rambarde. Face aux anges qui jouent de la trompette à l'entrée du grand thêâtre d'Oklahoma, l'effet de contre-plongée est encore accentué. Une dizaine de garçons regardent d'en bas ces femmes déguisées en anges et montées sur des piédestaux. Leurs regards à la fois intéressés et extérieurs démultiplient celui que Karl portait sur la statue de la Liberté avant son odyssée américaine. Arrivé au terme de sa quête, Karl s'intégrera brièvement à ce tableau allégorique. C'est là que, les yeux levés, il atteindra enfin pour quelques instants les hauteurs du bonheur.

La mise en scène de cette ascension est très élaborée. Karl escalade d'abord le podium sous le regard des garçons. Puis il s'avance sous le regard des femmes. Enfin un ange l'appelle: «Il leva les yeux

29. Vialatte commet ici l'erreur de «synonymiser», que Kundera reproche aux traducteurs. L'emploi de «mélancoliquement» au lieu de «tristement» gomme en effet la répétition du lexème «triste», caractéristique du texte original (Milan Kundera, "Une phrase», l'Infini, 35, 1991, p. 46).

30. Torton Beck, Op. cit., p. 125, n. 10. 
et se mit à rire, de surprise et de joie; c'était Fanny.» (L.311) Le lecteur ne connaît pas davantage Fanny qu'il ne connaît le signalement de Karl, mais l'entente profonde qui unit ensuite l'homme et la femme ne fait pas l'ombre d'un doute. Sous le couvert d'une simple scène de retrouvailles, les paroles et les gestes notés décrivent un coït qui vient contrebalancer le viol subi par Karl avant son départ pour l'Amérique. À la suite du regard, c'est la voix puis la main de Karl qui s'élevent vers Fanny. «Mais viens donc» (L.311), dit-elle alors, non sans écarter ses voiles pour découvrir un étroit escalier menant jusqu'à elle. Karl le gravit, tandis qu'elle s'écrie: «Plus lentement» (V.317) craignant une chute.... Enfin, il arrive sans encombre sur la demière marche et les deux se saluent, car l'enjeu apparent de la scène c'est de «se serrer la main». Après cela, Karl lève encore la main pour se prouver que Fanny est presque la plus haute parmi les femmes-anges du grand thêâtre. Puis elle lui prête sa trompette, qui remplace ici, en tant que symbole phallique, le glaive de la statue de la Liberté. Karl en joue si bien «à pleins poumons» que toutes les femmes l'écoutent à la ronde. «Tu es un artiste» (L.312) conclut alors son amie.

L'allégorie du grand théâtre permet d'amplifier et de développer largament l'euphorie liée au regard que Karl lève vers Fanny. Le parcours ascendant dessiné avec insistance se découvre bien sûr tout aussi bien dans les traductions que dans l'original, car l'image de l'ami, l'amant et l'artiste, fêté au sommet d'un piédestal, est assez éloquente en elle-même. Lorsque Kremer affirme que l'on ne trouve pas chez Kafka, comme chez Flaubert, par exemple, de stylisation de l'artiste et s'étonne d'une telle retenue, il fait sans doute abstraction d'un personnage comme Karl. Sa thèse selon laquelle l'érotisme de l'écriture serait extérieur à la jouissance du corps chez Kafka est nettement contredite par la scène des retrouvailles avec Fanny, car on y voit, bien au contraire, que la performance artistique coïncide avec la rencontre des corps. Dans un article récent, Kundera fait l'éloge des deux phrases du Château décrivant les coïts de K. et de Frieda. Son étude minutieuse de la façon dont les versions françaises déforment la rigueur et la beauté de ces phrases fait ressortir l'enjeu existentiel des longues métaphores qui, dans ce roman, figurent l'acte amoureux. À son avis, leur beauté exceptionnelle en dit long «sur l'importance de 
l'érotisme pour Kafkas ${ }^{31}$. Bien que l'exaltation de cette dimension du corps s'inscrive d'une tout autre façon dans le cadre allégorique du grand théâtre d'Oklahoma, l'enjeu existentiel n'y est pas moins présent, car, tout en réalisant ses plus hautes aspirations, Karl n'obtient pas la place d'acteur qu'il souhaite... Le sommet qu'il atteint se situe en marge des tribulations de l'embauche. Au-delà du podium des anges, qui n'est qu'un lieu de passage pour Karl, se trouve la dure réalité du champ de course.

En plus de l'axe vertical le long duquel les regards de Karl poursuivent le bonheur et le malheur, il y a l'axe horizontal sur lequel se joue une autre sorte de dialectique. Karl laisse volontiers filer son regard vers l'horizon, sans but précis, et découvre ainsi des lignes de réflexion. Puis, pour ne pas se perdre, il jette un regard à la ronde et se situe par rapport aux données concrètes de son entourage. Dès la première page du roman, ce double jeu du regard s'instaure. Lortholary dit très justement que le regard de Karl «erre» (L.17) au moment où celui-ci s'aperçoit qu'il a oublié son parapluie au fond du bateau, car über seinen Bekannten hinsehen (7) contient un élément d'indétermination qui se perd dans l'expression «jeter les yeux sur quelqu'un» choisie par Vialatte (V.12). Avant de partir à la recherche de ce parapluie, Karl repère les lieux. Pour überblickte schnell die Situation (8) Vialatte dit «examina encore l'endroit» (V.12), tandis que Lortholary propose «jeta un coup d'œil circulaire» (L.18). Quoi qu'il en soit, il s'agit d'un regard précis qui s'oppose au regard vague qui le précède. Ce type de regard circulaire sert parfois à prendre le pouls des gens qui observent Karl, mais il sert surtout à trouver des lignes de fuite $^{32}$. Karl a horreur de rester pris comme dans une souricière. Ses fuites effectives ou vellétaires sont au fond aussi importantes que les rejets qui structurent sa carrière.

La première fois qu'il cherche à s'échapper, il est coincé dans la cabine du soutier: Karl[...]sah sich um, wie er hinauskommen könnte

31. Milan Kundera, Op. cit., p. 49.

32. Gilles Deleuze et Félix Guattari étudient tout particulièrement cette configuration du désir dans Kafka. Pour une littérature mineure (Paris, Minuit, 1975, p. 26). 
(11). Voilà l'exemple type d'un verbe de perception suivi de wie et donc d'une difficulté de traduction. Il est bien clair que deux mouvements sont essentiels pour la fuite de Karl: regarder alentour et sortir. Mais la traduction ne peut pas tenir compte de ce double mouvement. Oserait-on dire «il regarde autour de lui comme pour sortir» en soulignant la finalité du regard, ou faut-il dédoubler ce regard en une composante physique et une composante intellectuelle? On dirait alors: «Il regarde autour de lui et se demande comment il pourrait sortir». Vialatte et Lortholary optent pour la transformation nominale: "chercher des yeux un passage» (V.15) ou encore «chercher des yeux une issue» (L.20). Le regard devient complément circonstanciel de manière d'une recherche tout au plus implicite dans le texte original. De même, l'action de sortir qui concerne le héros cède la place à des indications de lieu dont le texte original ne fait pas état. À cet égard, Jean-Pierre Demarche propose une solution plus juste en disant: «il chercha des yeux le moyen de s'échapper $»^{33}$.

Faute de pouvoir toujours s'échapper, Karl recherche les lieux qui permettent à son regard de vagabonder. Il aime tourner ses regards vers l'ailleurs. Sa place préférée est près de la fenêtre ou sur le balcon. On le retrouve dans cette posture chez son oncle, chez Pollunder, à l'Hôtel Occidental, chez Brunelda, dans le bureau dont il rêve et finalement dans le train qui emmène toute la troupe à Oklahoma. C'est là qu'il croit comprendre enfin les dimensions de l'Amérique en ne se lassant pas de regarder par la portière. C'est en laissant filer son regard vers l'horizon qu'il se retrouve lui-même.

Que révèle en définitive l'observation de la représentation romanesque de l'interaction telle que Kafka la pratique et telle que les traducteurs tentent de la rendre en français? Il faut reconnaitre d'abord que la syntaxe kafkaienne construit volontiers l'orchestration de l'interaction verbale et non verbale en coordonnant les données audibles et visibles. À la lecture de ces partitions, il n'y a souvent que l'ordre de la juxtaposition des éléments qui puisse suggérer une éventuelle logique

33. Franz Kafka, Der Heizer, présentation et notes de Jean-Pierre Demarche (Paris, éd. Marketing, 1991), p. 10. 
de la composition. L'ordre d'enchaînement est, en effet, presque toujours le même. Au discours, qui ne fait généralement pas l'objet de commentaires de la part du narrateur, s'ajoutent les mentions d'éléments communicatifs non verbaux, dont la qualité ou le sens peuvent être spécifiés ou commentés. On peut donc affirmer qu'en suivant les notations du narrateur le lecteur de Der Verschollene entend, voit, puis comprend les protagonistes des interactions décrites. Mais cette compréhension n'est jamais définitive, car elle dépend souvent d'un focalisateur peu fiable, Karl, qui ne connaît rien à l'Amérique!

Les traducteurs suivent bien sûr autant que faire se peut ce schéma de base, mais la réticence du français à la simple juxtaposition d'éléments hétérogènes entraîne souvent une syntaxe plus complexe qui homogénéise la représentation du processus interactif en liant plus explicitement ses divers registres les uns aux autres. Cela est très frappant dans la version de Vialatte. Ce l'est un peu moins dans celle de Lortholary qui présente cependant presque systématiquement l'élément non verbal coordonné sous la forme d'un gérondif qui le subordonne à l'acte de parole. Par ce fait, la représentation verbale du processus interactif se cristallise en suites de tableaux structurant l'enchaînement du discours kafkaien. Ce détail en rejoint un autre, beaucoup plus visible, dont Kundera s'inquiète à juste titre à propos du Chateau. Pourquoi les versions françaises ne respectent-elles pas le style fluide et continu de l'écriture kafkaïenne? Pourquoi une articulation graphique du texte découpé en de multiples paragraphes brise-t-elle le grand souffle des paragraphes infinis de $\mathrm{Kafka}^{34}$ ? Tout se passe comme si le rationalisme, dont la culture française se glorifie et qu'elle croit consubstantiel de sa langue à la suite de Rivarol, ne pouvait pas accueillir sans réserve la parataxe et les enchaînements de la prose kafkaïenne.

Par ailleurs, l'indication scrupuleuse du relativisme de la perception que l'on trouve dans Der Verschollene se perd un peu dans les versions qui ignorent parfois la modalité d'une comparaison ou privent carrément le focalisateur de son statut de sujet de la perception. Lorsque l'original ne fait que suivre le regard de Karl, les versions font

34. M. Kundera, Op. cit., pp. 51-52. 
alors «paraître» ou «apparaître» le monde. Ainsi, elles tendent à diminuer la vitalité du corps du disparu qui ne cesse pourtant de déchiffrer le monde et d'y chercher sa place. Si Kafka ne livre pas le signalement de son personnage, il expose par contre son corps aux multiples exigences de l'interaction sociale et lui fait arpenter du regard le monde qui façonne sa condition humaine.

\section{Université Laval}

- Ce travail a été rendu possible grâce à une subvention du Conseil de recherches en sciences humaines du Canada. Plusieurs analyses dont il rend compte sont basées sur une banque de données établie par Heidi Wagner et Sophie Bernier. Je tiens à les remercier ici toutes deux de leur précieuse collaboration. 